\title{
Cosmological neutrino mass constraint from the WiggleZ Dark Energy Survey
}

\author{
Signe Riemer-Sørensen* ${ }^{* \dagger}$ \\ University of Queensland \\ E-mail: Signedphysics.ua.edu.au
}

\begin{abstract}
The absolute neutrino mass scale is currently unknown, but can be constrained from cosmology. We use the large-scale structure information from the WiggleZ Dark Energy Survey to constrain the sum of neutrino masses. The WiggleZ high redshift star-forming blue galaxy sample is less sensitive to systematic effects from non-linear structure formation, pairwise galaxy velocities, redshift-space distortions, and galaxy bias than previous surveys. Through exhaustive tests using numerical dark-matter simulations of the WiggleZ survey, we demonstrate that at small scales common modelling approaches lead to systematic errors in the recovered cosmological parameters, and we use the simulations to calibrate a new non-linear fitting formula extending to small scales $\left(k=0.3 \mathrm{~h} \mathrm{Mpc}^{-1}\right)$. We obtain an upper limit on the sum of neutrino masses of $0.60 \mathrm{eV}(95 \%$ confidence) for WiggleZ+Wilkinson Microwave Anisotropy Probe. Combining with priors on the Hubble Parameter and the baryon acoustic oscillation scale gives an upper limit of $0.29 \mathrm{eV}$, which is the strongest neutrino mass constraint derived from spectroscopic galaxy redshift surveys.
\end{abstract}

36th International Conference on High Energy Physics

4-11 July 2012

Melbourne, Australia

* Speaker.

${ }^{\dagger}$ SRS thanks the organisers for financial support to participate in the conference. 


\section{Introduction}

In the Standard Model of particle physics the neutrinos are treated as exactly massless despite neutrino oscillation experiments having measured mass differences between the three species. No current experiment has the sensitivity to measure the absolute neutrino mass but it can be inferred from observations of the galaxy distribution today because massive neutrinos suppress the gravitational collapse of halos on small scales [U]]. The cosmic microwave background (CMB) provides an upper limit of $\sum m_{v}<1.3 \mathrm{eV}$ (all limits are 95\% confidence) [Q]. Combining with large-scale structure measurements such as the galaxy power spectrum [프, [25, 囵, 团], galaxy luminosity function [ [8], cluster mass function [[13, 四, or the baryon acoustic oscillations (BAO) [ए], 团] tightens the constraints to $\sum m_{v}<0.3 \mathrm{eV}$ by breaking parameter degeneracies. We use the galaxy power spectrum from the WiggleZ Dark Energy Survey to constrain the sum of neutrino masses.

\section{The WiggleZ Dark Energy Survey}

The WiggleZ Dark Energy Survey [[]] contains 238,000 galaxies with redshifts $z<1$ in a total volume of $1 \mathrm{Gpc}^{3}$. The Gigaparsec WiggleZ Survey (GiggleZ) simulations ${ }^{1}$ were designed to probe the low-mass haloes traced by WiggleZ galaxies, whilst providing an equivalent survey volume [ए6]. They provide a powerful means for testing and calibrating our modelling algorithms. WiggleZ has several potential advantages over previous surveys: 1) The neutrino suppression of the galaxy power spectrum is degenerate with effects from non-linear structure formation. Non-linearities increase with time so for the distant galaxies probed by WiggleZ, the contamination from nonlinearities is smaller than for previous surveys. This is illustrated in Fig. 1 where we show the ratio between a simulated WiggleZ power spectrum and the linear power spectrum for $z=0.2$ (dashed blue) and $z=0.6$ (solid black). For comparison we also show the ratio for simulated highly-biased massive haloes at $z=0.2$ (dotted red). 2) The relationship (bias) between the observed galaxy distribution and the dark matter distribution, which is influenced by massive neutrinos, depends on the observed galaxy type. Previous studies [155, [7]] measured red galaxies, which tend to cluster in the centers of dark matter halos, whereas the star-forming blue WiggleZ galaxies avoid the densest regions leading to a lower overall bias, reducing any systematics that could arise from the bias. The GiggleZ simulations show that over the range of scales and halo masses relevant for this analysis, the galaxy bias is scale-independent to within $1 \%$ whereas the neutrino scale dependent effect is of the order of $5 \%$ for $\sum m_{v}=0.3 \mathrm{eV}$ [园].

\section{Method and modelling}

Large-scale structure alone cannot determine all cosmological parameters, so we include data from WMAP (7 year). To compute the parameter likelihoods, we use importance sampling [12, [23] of the Markov Chain Monte Carlo (MCMC) chains ${ }^{2}$ from fitting to WMAP alone as well as to the chains combining WMAP with the BAO scale from SDSS Luminous Red Galaxies [प5] and a $H_{0}=$

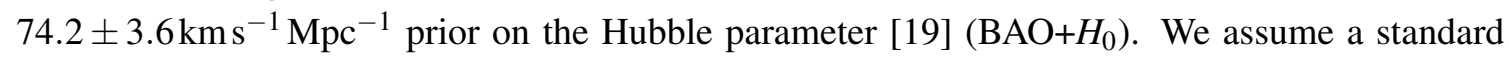

\footnotetext{
${ }^{1}$ With cosmological parameters $\Omega_{b}=0.049, \Omega_{m}=0.297, h=0.7, n_{s}=1.0, \sigma_{8}=0.8$ [B]]

${ }^{2}$ http: // lambda.gsfc.nasa.gov/product/map/dr4/parameters.cfm
} 

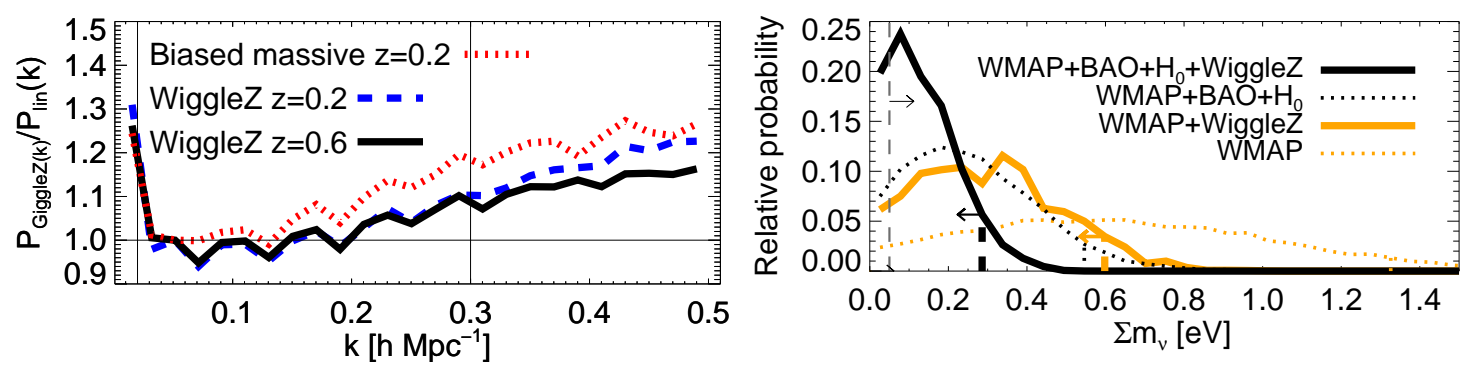

Figure 1: Left: The ratio between a simulated WiggleZ halo power spectrum and corresponding linear power spectrum. The vertical lines are our fitting range. Non-linear corrections are clearly less significant for the high-redshift, low-bias WiggleZ halos than at lower redshifts. Right: The relative probability distribution of $\sum m_{v}$ from fitting model F) with $k_{\max }=0.3 \mathrm{~h} \mathrm{Mpc}^{-1}$. The dashed grey line is the lower limit from oscillation experiments, and the vertical lines are $95 \%$ confidence upper limits.

flat $\Lambda \mathrm{CDM}$ cosmology with no time variation of $w$ and $N_{\mathrm{eff}}=3.04$, and fit over the parameters: $\Omega_{c}$ (cold dark matter density), $\Omega_{b}$ (baryon density), $\Omega_{\Lambda}$ (dark energy density), $\Omega_{v}$ (neutrino density), $h$ (Hubble parameter), $n_{s}$ (spectral index), $\Delta_{R}^{2}$ (amplitude of primordial density fluctuations).

Massive neutrinos suppress the power spectrum on all scales smaller than their free-streaming length at the time the neutrinos become non-relativistic. For $\sum m_{v}=0.3 \mathrm{eV}$ the most significant suppression happens for $k=0.3-1.3 \mathrm{~h} \mathrm{Mpc}^{-1}$, but the $k$-dependence of the suppression is more pronounced for $k<0.3 \mathrm{~h} \mathrm{Mpc}^{-1}$ and consequently easier to disentangle from other cosmological parameters and systematics [प], []]. At low redshift structure formation is no longer linear for $k<0.1 \mathrm{hpc}^{-1}$ [ㄴ], 24, [20, 2]], and simulations show that redshift-space distortions become $k$ dependent at low redshift and consequently are degenerate with neutrino mass [ [U, [4]]. By fitting to simulated power spectra from GiggleZ, we tested six different models for the non-linear effects: A) Linear structure formation with linear bias. B) Non-linear structure formation with linear bias. C) Non-linear structure formation and fitting formula for redshift space distortions and pairwise galaxy velocities. D) Same as C) but with zero pairwise velocities. E) Non-linear structure formation with pairwise galaxy velocity damping. F) Simulation calibrated model.

The details of the models are given in Riemer-Sørensen et al. 2012 [एष] and they are shown in Fig. $\square$ for a fixed cosmology. They are similar at low $k$, where the large-scale clustering is linear and the theory is robust, but for $k>0.2 \mathrm{~h} \mathrm{Mpc}^{-1}$ the differences increase. The right part of Fig. shows the the reduced $\chi^{2}$ from fitting each of the models to simulated power spectra. The upper panel is the $\chi^{2}$ for the simulation parameters. It is clear that model B), C), E) are unable to provide a good fit for the correct parameter values for $k_{\max }>0.2 \mathrm{~h} \mathrm{Mpc}^{-1}$. The lower panel shows the distance in $\chi^{2}$ between the best fitting parameters and the simulation ones, which is a measure of how well the models recover the simulated parameter values. It is clear that at small scales models A)-E) are insufficient, and the complexity of model F) is necessary.

Model F: The non-linear effects are present in an $N$-body simulation for a fiducial cosmology and can be implemented following the approach of Reid et al. 2010 [ए7]. For each trial cosmology:

$$
P_{\mathrm{gal}}^{\text {trial }}(k)=b^{2} P_{\mathrm{hf}, \mathrm{nw}}^{\mathrm{trial}}(k) \frac{P_{\mathrm{dmped}}^{\text {trial }}(k)}{P_{\mathrm{nw}}^{\text {trial }}(k)} \frac{P_{\mathrm{GiggleZ}}^{\mathrm{fid}}(k)}{P_{\mathrm{hf}, \mathrm{nw}}^{\mathrm{fid}}(k)},
$$



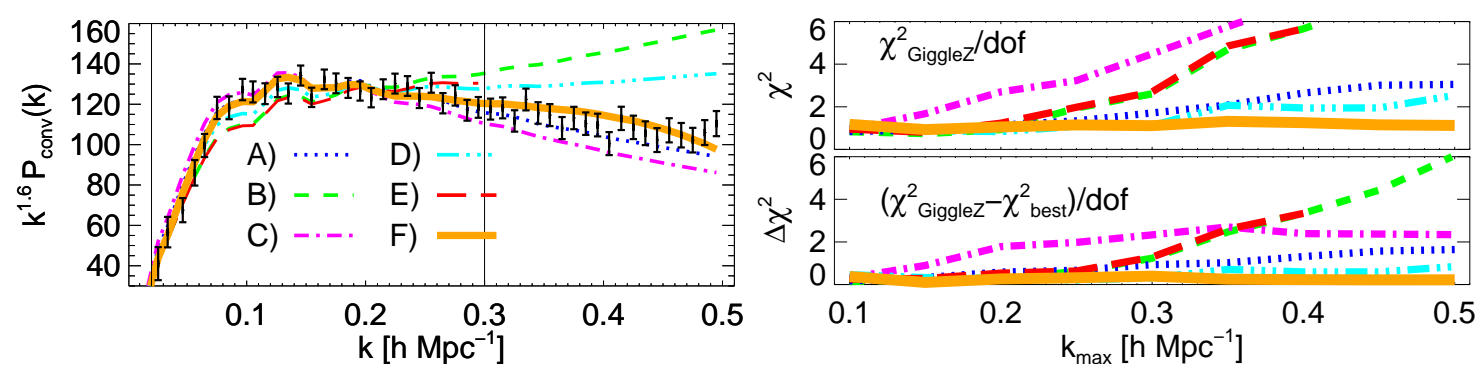

Figure 2: Left: The observed WiggleZ power spectrum, and the six models for the best fit cosmology of model F). The vertical lines are our fitting range. The divergence between the models at large $k$ is clear and demonstrates the importance of careful modeling. Left upper: Reduced $\chi^{2}$ of models A)-F) fitted to the $N$-body simulation halo catalogue for the GiggleZ fiducial cosmology values. In absence of systematic errors the models should recover the input cosmology with $\chi^{2} / \mathrm{dof}=1$. Left lower: Difference in reduced $\chi^{2}$ values when using the GiggleZ fiducial cosmological parameters and the best fit values.

where $P_{\text {damped }}^{\text {trial }}(k)=P_{\text {lin }}^{\text {trial }}(k) f_{\text {damp }}(k)+P_{\text {nw }}^{\text {trial }}(k)\left(1-f_{\text {damp }}(k)\right)$ and $f_{\text {damp }}(k)=\exp \left(-\left(k \sigma_{v}\right)^{2}\right)$ with $\sigma_{v}$ given by $\sigma_{v}^{2}=1 /\left(6 \pi^{2}\right) \int d k^{\prime} P_{\text {lin }}\left(k^{\prime}\right) . P_{\text {GiggleZ }}^{\text {fid }}(k)$ is a $5^{\text {th }}$ order polynomial fit to the power spectrum of a set of halos in the GiggleZ simulations chosen to match the clustering amplitude of WiggleZ galaxies. $P_{\text {lin }}(k)$ and $P_{\mathrm{nw}}(k)$ are the linear and non-linear power spectra from $\mathrm{CAMB}^{3}$, where the latter implements Halofit. [[22] $P_{\mathrm{nw}}$ and $P_{\mathrm{hf}, \mathrm{nw}}$ are the same power spectra but without the acoustic peaks. The factor of $b^{2}$ in Eqn. 3 i. is related to galaxy bias. The second factor represents the smooth power spectrum of the trial cosmology. The third factor defines the acoustic peaks and their broadening caused by the bulk-flow motion of galaxies from their initial positions in the density field, and the fourth factor describes all additional non-linear effects in the $N$-body simulation.

Throughout the analysis we have fixed the lower limit to be $k_{\min }=0.02 \mathrm{~h} \mathrm{Mpc}^{-1}$, which corresponds to the largest modes observed in each of the WiggleZ regions and we present all results as a function of $k_{\max }$.

\section{Results and discussion}

When fitting model F) to the observed WiggleZ power spectra we obtain a limit of $\sum m_{v}<$ $0.60 \mathrm{eV}$ for WMAP+WiggleZ with $k_{\max }=0.3 \mathrm{~h} \mathrm{Mpc}^{-1}$. Combining with $\mathrm{BAO}+H_{0}$ reduces the uncertainty in $\Omega_{m}$ and $H_{0}$, leading to a stronger neutrino mass constraints of $\sum m_{v}<0.29 \mathrm{eV}$.

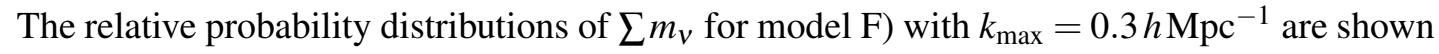
in Fig. $\mathbb{~}$. It is clear how adding WiggleZ data to the analysis narrows the distributions (dotted to solid) both with (orange) and without (black) the inclusion of BAO+H$H_{0}$. This is the strongest neutrino mass limit so far derived from spectroscopic redshift galaxy surveys. The advantages of WiggleZ are a higher redshift for which the structure formation is linear to smaller scales, and a simple galaxy bias for the strongly star-forming blue emission line galaxies.

Our result is comparable to that obtained using independent methods [25], [1, [1], 四], but with different systematics. Since the data sets are all independent, they can potentially be combined in the future to provide even stronger constraints. This is particularly interesting in light of recent

\footnotetext{
$3_{\text {http: }: / / \text { camb.info }}$
} 
results [Q, [0] that hint at the existence of additional neutrino species $\left(N_{\text {eff }}>3.04\right)$. Allowing for additional neutrino species degrades the constraining power of large scale clustering alone, and the combination of $N_{\text {eff }}$ and $\sum m_{v}$ is therefore poorly constrained with current data.

In the future, galaxy surveys such as the Baryon Acoustic Oscillation Survey, Dark Energy Survey and Euclid will be far more sensitive, however, as demonstrated in this paper, the small details of the modelling of non-linear effects become very important, so robust modelling either theoretically or calibrated to simulations with massive neutrinos will be necessary.

\section{References}

[1] B. A. Benson, T. de Haan, J. P. Dudley, et al., Cosmological Constraints from Sunyaev-Zel'dovich-Selected Clusters with X-ray Observations in the First 178 Square Degrees of the South Pole Telescope Survey, ArXiv:1112.5435 (2011).

[2] S. Bird, M. Viel, and M. G. Haehnelt, Massive Neutrinos and the Non-linear Matter Power Spectrum, ArXiv:1109.4416 (2011).

[3] C. Blake, S. Brough, M. Colless, et al., The WiggleZ Dark Energy Survey: the selection function and $z$ $=0.6$ galaxy power spectrum, Mon. Not. Roy. Astron. Soc. 406 (2010), 803-821.

[4] R. de Putter, O. Mena, E. Giusarma, et al., New Neutrino Mass Bounds from Sloan Digital Sky Survey III Data Release 8 Photometric Luminous Galaxies, ArXiv:1201.1909 (2012).

[5] M. J. Drinkwater, R. J. Jurek, C. Blake, et al., The WiggleZ Dark Energy Survey: survey design and first data release, Mon. Not. Roy. Astron. Soc. 401 (2010), 1429-1452.

[6] M. C. Gonzalez-Garcia, M. Maltoni, and J. Salvado, Updated global fit to three neutrino mixing: status of the hints of $\theta_{13}>0$, Journal of High Energy Physics 4 (2010), 56.

[7] E. Jennings, C. M. Baugh, and S. Pascoli, Modelling redshift space distortions in hierarchical cosmologies, Mon. Not. Roy. Astron. Soc. (2010), 1572.

[8] C. Jose et al., Weighing neutrinos using high redshift galaxy luminosity functions, Phys. Rev. D83 (2011), no. 12, 123518.

[9] E. Komatsu, K. M. Smith, J. Dunkley, et al., Seven-year Wilkinson Microwave Anisotropy Probe (WMAP) Observations: Cosmological Interpretation, The Astrophysical Journal Supplement 192 (2011), 18.

[10] J. Kopp, M. Maltoni, and T. Schwetz, Are There Sterile Neutrinos at the eV Scale?, Physical Review Letters 107 (2011), no. 9, 091801.

[11] J. Lesgourgues and S. Pastor, Massive neutrinos and cosmology, Physics Reports 429 (2006), 307-379.

[12] A. Lewis and S. Bridle, Cosmological parameters from CMB and other data: A Monte Carlo approach, Phys. Rev. D66 (2002), no. 10, 103511.

[13] A. Mantz, S. W. Allen, and D. Rapetti, The observed growth of massive galaxy clusters - IV. Robust constraints on neutrino properties, Mon. Not. Roy. Astron. Soc. 406 (2010), 1805-1814.

[14] F. Marulli, C. Carbone, M. Viel, et al., Effects of massive neutrinos on the large-scale structure of the Universe, Mon. Not. Roy. Astron. Soc. 418 (2011), 346-356.

[15] W. J. Percival, B. A. Reid, D. J. Eisenstein, et al., Baryon acoustic oscillations in the Sloan Digital Sky Survey Data Release 7 galaxy sample, Mon. Not. Roy. Astron. Soc. 401 (2010), 2148-2168. 
[16] G. B. Poole and others, In preparation (2012).

[17] B. A. Reid, L. Verde, R. Jimenez, et al., Robust neutrino constraints by combining low redshift observations with the CMB, Journal of Cosmology and Astro-Particle Physics 1 (2010), 3.

[18] S. Riemer-Sørensen, C. Blake, D. Parkinson, et al., WiggleZ Dark Energy Survey: Cosmological neutrino mass constraint from blue high-redshift galaxies, Phys. Rev. D85 (2012), no. 8, 081101.

[19] A. G. Riess, L. Macri, S. Casertano, et al., A Redetermination of the Hubble Constant with the Hubble Space Telescope from a Differential Distance Ladder, ApJ699 (2009), 539-563.

[20] S. Saito, M. Takada, and A. Taruya, Impact of Massive Neutrinos on the Nonlinear Matter Power Spectrum, Physical Review Letters 100 (2008), no. 19, 191301.

[21] R. Scoccimarro, Redshift-space distortions, pairwise velocities, and nonlinearities, Phys. Rev. D70 (2004), no. 8, 083007.

[22] R. E. Smith, J. A. Peacock, A. Jenkins, et al., Stable clustering, the halo model and non-linear cosmological power spectra, Mon. Not. Roy. Astron. Soc. 341 (2003), 1311-1332.

[23] M. E. C. Swanson, W. J. Percival, and O. Lahav, Neutrino masses from clustering of red and blue galaxies: a test of astrophysical uncertainties, Mon. Not. Roy. Astron. Soc. 409 (2010), 1100-1112.

[24] M. Takada, E. Komatsu, and T. Futamase, Cosmology with high-redshift galaxy survey: Neutrino mass and inflation, Phys. Rev. D73 (2006), no. 8, 083520.

[25] S. A. Thomas, F. B. Abdalla, and O. Lahav, Upper Bound of $0.28 \mathrm{eV}$ on Neutrino Masses from the Largest Photometric Redshift Survey, Physical Review Letters 105 (2010), no. 3, 031301. 